\title{
Laser Systems for Orbital Debris Removal
}

\section{LLNL-PROC-423323}

A. M. Rubenchik, C. P. Barty, R. J. Beach, A. C. Erlandson, J. A. Caird

February 8, 2010

HPLA 2010

Santa Fe, NM, United States

April 18, 2010 through April 22, 2010 
This document was prepared as an account of work sponsored by an agency of the United States government. Neither the United States government nor Lawrence Livermore National Security, LLC, nor any of their employees makes any warranty, expressed or implied, or assumes any legal liability or responsibility for the accuracy, completeness, or usefulness of any information, apparatus, product, or process disclosed, or represents that its use would not infringe privately owned rights. Reference herein to any specific commercial product, process, or service by trade name, trademark, manufacturer, or otherwise does not necessarily constitute or imply its endorsement, recommendation, or favoring by the United States government or Lawrence Livermore National Security, LLC. The views and opinions of authors expressed herein do not necessarily state or reflect those of the United States government or Lawrence Livermore National Security, LLC, and shall not be used for advertising or product endorsement purposes. 


\title{
Laser Systems for Orbital Debris Removal
}

\author{
A.M. Rubenchik, C.P.J. Barty, R.J. Beach, A.C. Erlandson, and J.A. Caird \\ Lawrence Livermore National Laboratory, 7000 East Ave, Livermore, CA 94550, USA
}

\begin{abstract}
The use of a ground based laser for space debris cleaning was investigated by the ORION project in 1996. Since that study the greatest technological advance in the development of high energy pulsed laser systems has taken place within the NIF project at LLNL. The proposed next laser system to follow the NIF at LLNL will be a high rep rate version of the NIF based on diode-pumping rather than flashlamp excitation; the so called "LIFE" laser system. Because a single "LIFE" beamline could be built up in a few year time frame, and has performance characteristics relevant to the space debris clearing problem, such a beamline could enable a near term demonstration of space debris cleaning. Moreover, the specifics of debris cleaning make it possible to simplify the LIFE laser beyond what is required for a fusion drive laser, and so substantially reduce its cost. Starting with the requirements for laser intensity on the target, and then considering beam delivery, we will flow back the laser requirements needed for space debris cleaning. Using these derived requirements we will then optimize the pulse duration, the operational regime, and the output pulse energy of the laser with a focus of simplifying its overall design. Anticipated simplifications include operation in the heat capacity regime, eliminating cooling requirements on the laser gain slabs, and relaxing B-integral and birefrigence requirements.
\end{abstract}

Keywords: space debris, laser, pulsed lasers, debris cleaning

PACS: 42.60.By, 42.55.Xi

\section{INTRODUCTION}

The proliferation of satellites in Earth orbit, increasing both in number and value makes the problem of collision with orbital debris very real. One of the most reasonable solutions for the problem is debris removal with the help of a ground based pulsed laser. In this approach laser pulses ablate debris material, change the velocity and move the debris to the lower orbit where natural burn-up takes place. This method of debris removal was analyzed by the "Orion" project [1] where the requirement for the laser and optical and tracking systems were summarized. Two things have changed since project completion. The danger of valuable asset damage is so serious that governments may be ready to spend money on orbital debris removal. Secondly, a significant advance in powerful pulsed laser technology took place, mainly at Lawrence Livermore National Laboratory (LLNL), with completion of the National Ignition Facility (NIF) project $[2,3]$. An aerial photograph of the NIF is shown in Figure 1 with the roof of the building removed, and an engineering rendering of the laser system inserted. We will show that laser systems designed for inertial confinement fusion applications are a perfect fit for the orbital debris removal application.

We start the analysis with requirements for the laser pulse on the target. Then we discuss the propagation and focusing to more completely define requirements for the laser. Based on this we specify a range of parameters for laser operation and discuss how the laser design can be simplified. Finally, we present a schematic diagram of the laser along with estimates of its performance specifications, size and cost. 


\section{INTERACTION OF LASER RADIATION WITH DEBRIS}

High intensity pulsed laser radiation striking the debris vaporizes surface material, creating a recoil impulse that changes the debris velocity. It is clear that for fixed pulse duration an optimum laser intensity exists. For low intensity the target temperature and ablation will be

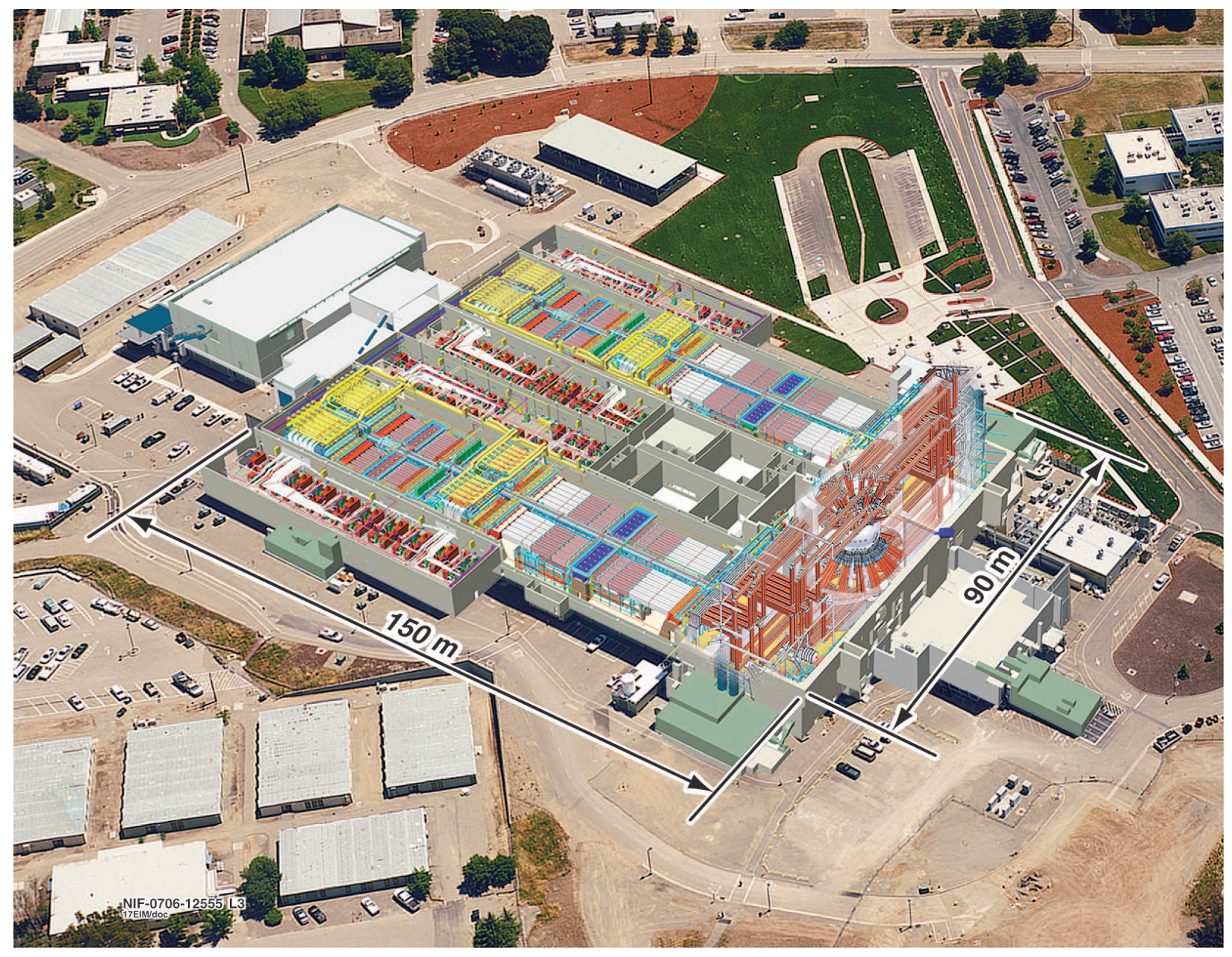

FIGURE 1. NIF is approximately $150 \mathrm{~m} \times 90 \mathrm{~m}$ and seven stories tall. The two laser bays are shown on the upperleft portion of the figure. The switchyard (in red) is shown on the lower-right side, as is the spherical target chamber (in silver), into which the 192 beamlines converge.

low and the recoil impulse is small. For high intensities a lot of energy will be wasted on material ionization. A crucial parameter for pulsed laser debris removal is the coupling coefficient $\mathrm{C}_{\mathrm{m}}$, the ratio of momentum imparted to the target to the incident laser energy, $\mathrm{C}_{\mathrm{m}}=$ $\triangle \mathrm{P} / \mathrm{E}$. A review of data on $\mathrm{C}_{\mathrm{m}}$ dependence on intensity for different materials is presented by Phipps [4]. The experimental data from different groups demonstrates that for a broad range of wavelength, pulse duration and pulse energy the coupling coefficient maximum is reached at intensity

$$
I_{m}=\frac{2.5 \mathrm{GW} / \mathrm{cm}^{2}}{\sqrt{\tau(\mathrm{ns})}}
$$


where $t(n s)$ is the pulse duration in nanoseconds.

The numerical coefficient is valid for Al alloys but does not change very much for different materials and wavelengths. The temporal dependence indicates that the surface temperature and ablation is controlled by the thermal flux from the surface. As a function of laser intensity, $\mathrm{C}_{\mathrm{m}}$ is peaked not far from the vaporization threshold where plasma starts to be generated and absorptivity increases rapidly, which explains weak sensitivity to target material. Typical values of $\mathrm{C}_{\mathrm{m}}$ are 1-10 dyne/W and depend weakly on material [4]. The coupling coefficient drops sharply at $\mathrm{I}<\mathrm{I}_{\mathrm{m}}$ and gradually decreases as

$\mathrm{C}_{\mathrm{m}} \sim\left(\mathrm{I}_{\mathrm{m}} / \mathrm{I}\right)^{1 / 3}$ at $\mathrm{I}>\mathrm{I}_{\mathrm{m}}$.

For pulse fluence corresponding to the optimal coupling we have

$$
\mathrm{F}=\mathrm{I}_{\mathrm{m}} \tau=2.5 \sqrt{\tau(\mathrm{ns})} \mathrm{J} / \mathrm{cm}^{2}
$$

Consider as representative debris a chunk of aluminum with size $\sim 10 \mathrm{~cm}$; weight $\sim 70 \mathrm{gm}$ with orbital altitude $\sim 500 \mathrm{~km}$. To change the orbit to an elliptical one with perigee $100 \mathrm{~km}$ one must produce a velocity change of $\Delta v=115 \mathrm{~m} / \mathrm{sec}$. [5] With an optimum coupling coefficient for $\mathrm{Al}, \mathrm{C}_{\mathrm{m}} \sim 6$ dyne/W, the energy to change the orbit $\mathrm{E} \sim \mathrm{m} \Delta \mathrm{v} / \mathrm{C}_{\mathrm{m}}=134 \mathrm{~kJ}$. For optimal fluence (3) and chunk area $\mathrm{S} \sim 100 \mathrm{~cm}^{2}$ one needs about E/FS $\sim 536 / \sqrt{\tau(\mathrm{ns})}$ shots to de-orbit the chunk. With a 5 ns pulse duration that amounts to $\sim 240$ pulses.

\section{OPTICAL SYSTEM}

Debris orbiting the Earth at altitude $\mathrm{h}$ becomes visible to the ground laser at distance $\mathrm{d} \sim$ $\sqrt{ } 2 \mathrm{Rh}$, where $\mathrm{R}$ is the Earth's radius. $\mathrm{d} \sim 2600 \mathrm{~km}$ for $\mathrm{h} \sim 500 \mathrm{~km}$. The time for this debris to travel from the horizon to the laser zenith is about 5.8 minutes. It is clear that there is an optimal angle to engage the debris. If the target is at the laser zenith - a laser pulse can only add a vertical component to the target velocity, which is not beneficial. When the target is on the horizon the distance is substantial and propagation through the atmosphere is long at low altitude with all possible detrimental effects. We believe the optimal angle for laser engagement is between 30 and 45 degrees from the horizon and the time interval for interaction with the target is about 45 seconds. For estimation consider the distance to the target $\mathrm{L} \sim 800 \mathrm{~km}$.

The diffraction limited spot radius at distance L made by mirror with diameter D

$$
r=\frac{2 \lambda L}{\pi D}
$$

For $1 \mu \mathrm{m}$ light, $\mathrm{L} \sim 800 \mathrm{~km}$ and $\mathrm{D}=3 \mathrm{~m}$ we have the diffraction limited spot size of $\mathrm{r} \sim 18 \mathrm{~cm}$. The total laser energy corresponding to the optimal fluence in the spot with radius $\mathrm{r}$ is $\mathrm{E} \sim \pi \mathrm{r}^{2} \mathrm{~F}$ $\sim 2.5 \mathrm{~kJ} \sqrt{\tau(\mathrm{ns})}$. Note that the required pulse energy is inversely proportional to the director mirror area and the square of the distance to the target. For pulse duration $\sim 5 \mathrm{~ns}$ the required pulse energy is about $6 \mathrm{~kJ}$. 
The above estimates were presented for 1 micron radiation. Conversion to the second and third harmonics can improve the focusing of laser radiation and reduce the required pulse energy. It is not clear, however, that the loss of energy in the frequency conversion process, and the difficulty of pointing the smaller spots on a very small target would justify complication of the laser design to produce the harmonic conversion.

The output pulse energy of laser systems drops sharply for pulses shorter than $1 \mathrm{~ns}$. Debris removal with very long pulses requires too much energy per pulse. A pulse duration of a few nanoseconds, therefore, looks optimal for a debris removal system. This pulse energy estimate ( $\sim 6 \mathrm{~kJ}$ for $5 \mathrm{~ns}$ pulse duration) is a lower bound. We must also take into account losses in the beam director, propagation in the atmosphere, and imperfect beam quality.

In addition, the above estimates were done for the normal incidence on a flat surface. Generally, the debris has a rugged shape and can rotate. As a result, it will be safer to have about $18 \mathrm{~kJ}$ per $\sim 5 \mathrm{~ns}$ pulse. A laser system with $15 \mathrm{~kJ}$ and a pulse duration of $10 \mathrm{~ns}$ was suggested by the Orion project [1].

For the powerful laser system needed for inertial confinement fusion the pulse duration is determined by the requirements of target compression and for modern targets is about a few nanoseconds. The pulse energy for a NIF beam-line is about $20 \mathrm{~kJ}$ at 1 micron. Therefore, we see a high synergy of requirements for laser systems for both problems.

\section{LASER SYSTEM}

Individual beamlines of the NIF have produced pulses in excess of $20 \mathrm{~kJ}$ at the 1 micron wavelength [2]. The pulse repetition rate at the NIF, however, is limited to a few shots per day. To enable economical production of fusion energy the repetition rate of the system must be increased to several pulses per second.

As part of our effort to develop laser technology for the inertial fusion energy application we built the Mercury laser system. Mercury uses an aperture-scalable laser architecture, and operates at a repetition rate of $10 \mathrm{~Hz}$ [6]. The primary advances demonstrated by Mercury include pumping by laser diode arrays to increase laser efficiency, and high speed helium gas cooling for heat removal to enable high average power operation.

In parallel with Mercury laser development, we created the preliminary conceptual design of a laser beamline for the Laser Inertial Fusion Energy (LIFE) application as shown in Figure 2 [7]. That system produces $12.5 \mathrm{~kJ}$ of $1 \mu \mathrm{m}$ output energy continuously at a repetition rate of 13.3 Hz. For the orbital debris removal application, however, a simplified and lower cost laser design is possible. This is because neither harmonic conversion nor continuous operation will be required. 


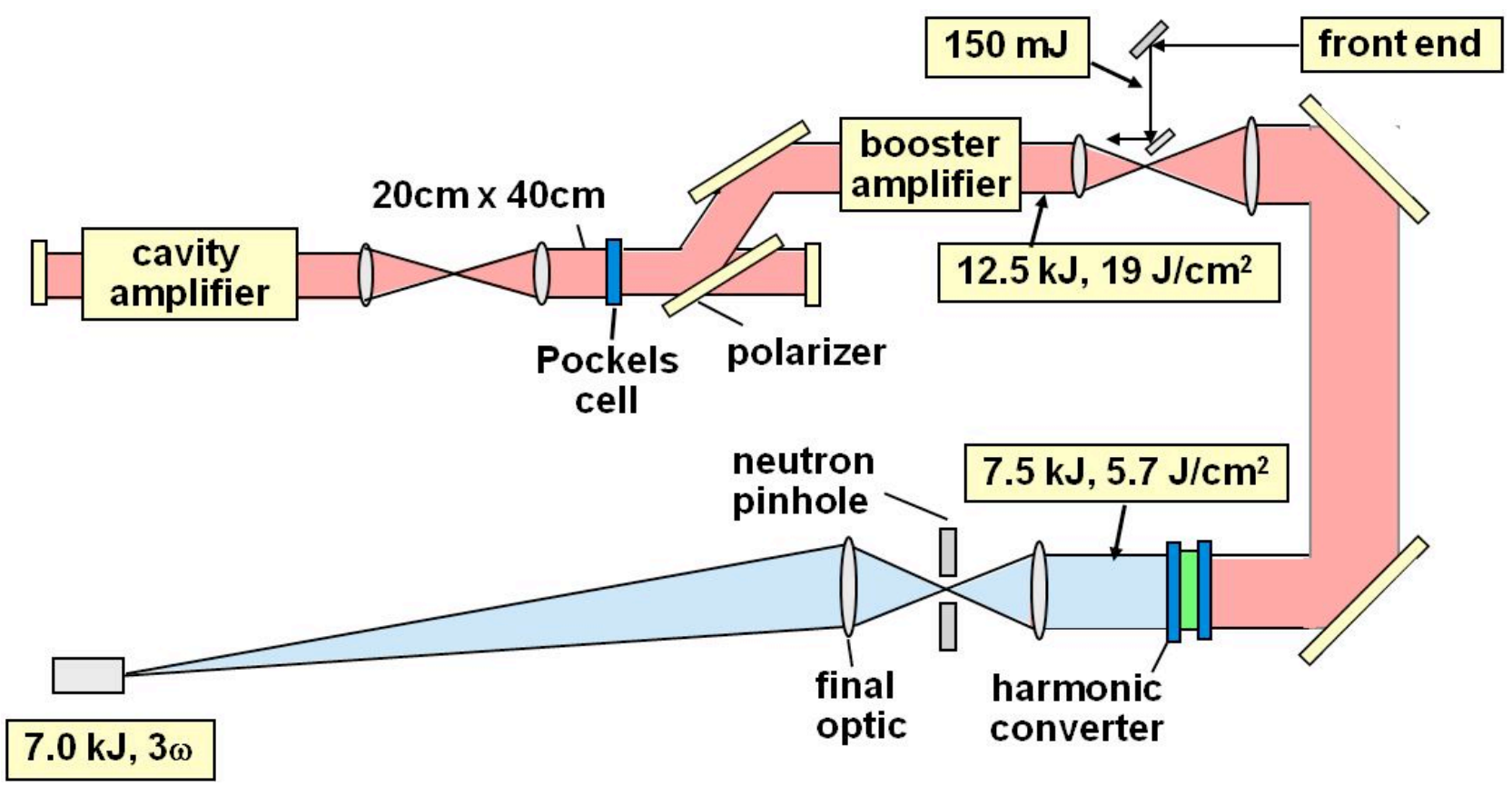

FIGURE 2. Schematic of the conceptual design of a diode pumped LIFE laser beam-line.

We demonstrated above that effective orbital debris removal can be accomplished with a few hundred pulses per target at similar energy levels. It will be possible to wait several minutes before engaging the next target. If the temperature increase at the end of a pulse train is within the operation range we can minimize the cooling system requirements, as in "heat capacity" laser systems [8].

For orbital debris removal the electrical efficiency of the laser is not a significant concern, so the pump diodes can be replaced by far less expensive flashlamps. Finally, since there is no need to protect the laser system from target back-reflections, the gain isolation system can be simplified so that a full aperture Pockels cell is not required. A simplified design schematic is shown in Figure 3. In this configuration the booster amplifier provides two additional passes of gain so that the required front end energy drops from $150 \mathrm{~mJ}$ to just $10 \mathrm{~mJ}$. To produce total energy of $\sim 20 \mathrm{~kJ}$, pulses from two such beamlines with $\sim 20-\mathrm{cm} \mathrm{x} 40-\mathrm{cm}$ apertures and with polarizations orthogonal to each other could be combined incoherently at a polarizer, then delivered to a common beam director. Alternatively, the $20-\mathrm{kJ}$ pulse energy could be produced using a single beamline, provided the aperture is scaled up to $40 \mathrm{~cm} \times 40 \mathrm{~cm}$. An engineering study would need to be undertaken to evaluate differences in cost and performance of these concepts.

This work performed under the auspices of the U.S. Department of Energy by Lawrence Livermore National Laboratory under Contract DE-AC52-07NA27344. 


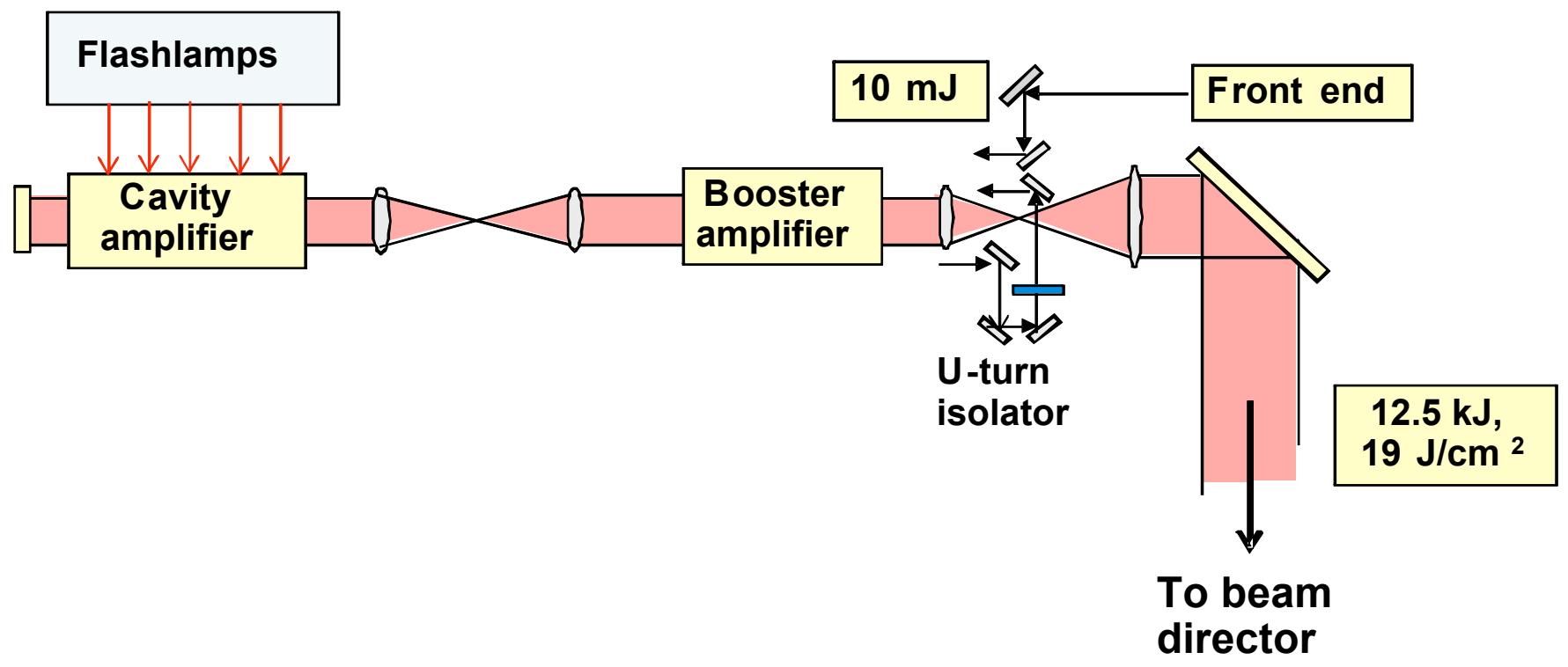

FIGURE 3. Schematic of a simplified laser design for orbital debris removal. 


\section{REFERENCES}

1. J. Campbell, Project ORION: Orbital Debris Removal Using Ground-Based Sensors and Lasers, NASA Technical Memorandum 108522 (1996).

2. C. Haynam, et. al., "National Ignition Facility laser performance status," Appl. Opt., $\underline{\mathbf{4 6}}, 3276-$ 3303 (2007).

3. C. Haynam, et. al., "The National Ignition Facility 192 Beam $3 \omega$ Laser Performance Status," paper 5.0.4, presented at IFSA, San Francisco, Sep. 11, 2009.

4. C. Phipps, et. al., "Impulse coupling to targets in vacuum by $\mathrm{KrF}, \mathrm{HF}$, and $\mathrm{CO}_{2}$ single-pulse lasers,” J. Appl. Phys., 뚜,1083-1096 (1988).

5. W. Schall, "Removal of small space debris with orbiting lasers," SPIE, $\underline{\mathbf{3 3 4 3}}, 564-574$, (1998).

6. A. Bayramian, et. al., "The Mercury project: A high average power, gas-cooled laser for inertial fusion energy development," Fus. Sci. and Tech., 프, 383-387 (2007).

7. J. Caird, et. al., "Nd:glass laser design for laser ICF fission energy (LIFE)," Fus. Sci. and Tech., 吕, 607-617 (2009).

8. G. Albrecht, et. al., "Solid state heat capacity disk laser," Laser and Particle Beams, $\underline{\mathbf{1 6}}$, 605625 (1998). 